\title{
Capital humano. La educación en Ecuador y su rentabilidad
}

\author{
URL: http://revistas.uta.edu.ec/erevista/index.php/bcoyu/article/view/690～Dol: http://dx.doi.org/10.31164/bcoyu.21.2019.690
}

\section{Bryan Espinoza-Estrella1; Ramiro Villarruel-Meythaler ${ }^{2}$}

Fecha de recepción: 27 de febrero de 2019

\section{Resumen}

Desde el año 2008 una serie de reformas laborales han sido implementadas en Ecuador bajo el principio de la mejora de las condiciones de vida del trabajador. Incluso, el sector de la educación fue también reestructurado para alcanzar mayores niveles de cobertura y calidad, y, junto con ello, los ingresos laborales se incrementaron dado este nuevo marco jurídico. Es así como, dentro de este contexto de reformas legales, el presente artículo analiza la rentabilidad de la educación y de sus diferentes niveles de instrucción educativa a través del desarrollo de modelos econométricos de regresión.

Palabras clave: Rentabilidad de la educación; reforma salarial; capital humano; modelos econométricos.

\section{Abstract}

Since 2008, a series of labor reforms have been implemented in Ecuador under the principle of improving the living conditions of the worker. Even the education sector was also restructured to achieve higher levels of coverage and quality; besides, labor income increased due to this new legal framework. Thus, within this context of legal reforms, this article analyzes the profitability of education and its different levels of education through the development of regression econometric models.

Keywords: Profitability of education; wage reform; human capital, econometric models.

\section{Introducción}

Para aquellos ajenos a la realidad ecuatoriana, previo a entender acerca de la rentabilidad de la educación y de sus diferentes niveles, hay que conocer las reformas que se han dado en el país. Sobre estas reformas se buscó incrementar la remuneración salarial y, por tanto, la calidad de vida del trabajador. En marzo de 2008 entró en vigencia el mandato ocho promulgado por la Asamblea Constituyente en el que se prohibió la tercerización y la intermediación laboral, se eliminó la contratación por horas, se estipuló que bajo ningún caso se suscribirán contratos con una remuneración inferior a la básica mínima unificada o a los mínimos sectoriales y, entre otras, se garantizaron todos los beneficios de ley, afiliación al Instituto Ecuatoriano de Seguridad Social -IESS- y demás derechos consagrados en la constitución (Asamblea Constituyente, 2008a). De hecho, en la carta magna de 2008 se planteó que la remuneración deberá cubrir al menos las necesidades básicas y, en principio, será revisada de manera anual con carácter progresivo (Asamblea Constituyente, 2008b).

Así entonces, hubo varias revisiones del Salario Básico Unificado -SBU-, que según el Código de Trabajo (Congreso Nacional, 2005), debe tender al costo de la canasta familiar básica (Art. 81); incluso, dentro de las revisiones, si el Consejo Nacional de Trabajo y Salarios -CNTSno llegase a un acuerdo se contemplaría un incremento equivalente al Índice de Precios al Consumidor -IPC- pero de manera proyectada (Art. 118). Se recalca que dicho consejo se levanta como un organismo técnico del Ministerio de Trabajo -MDT-, el cual, por consenso, definió que el SBU fuese de 386 dólares para el año 2018, es decir, planteó un incremento de once dólares en relación al año anterior en el que se consideró el 0,27\% de inflación. Por derrame, los sueldos tanto en el sector público y privado se fijaron entonces bajo este principio de mejora y de justicia hacia el trabajador. De esta manera, el MDT planteó a partir del primero de enero del año 2018 los salarios y tarifas mínimas sectoriales a nivel nacional, y los que recibirán los trabajadores privados amparados por el Código de Trabajo (Ministerio de Trabajo, 2017).
Fecha de aceptación: 22 de mayo de 2019

Dentro del área pública administrativa, la revisión de las remuneraciones se la hace a partir de la Ley Orgánica de Servicio Público LOSEP- (Asamblea Nacional, 2015) y del Código de Trabajo. EI MDT ya propuso los salarios para el año 2018 según los diferentes grupos ocasionales, aunque estos salarios se han mantenido vigentes desde el año 2012 (Ministerio de Trabajo, 2017). Entre estos grupos se detallan a los servidores públicos de servicios 1 y 2 , servidores públicos de apoyo del 1 al 4 y servidores públicos del 1 al 14; sueldos que en conjunto van desde los 374 a 3.542 dólares. Sobre las remuneraciones e ingresos complementarios tanto de las fuerzas armadas como de la policía nacional, se encuentran sujetos a lo establecido por el MDT según la misma LOSEP (Arts. 3, 51 y 102). Sobre este último marco, el MDT a través de su resolución MRL ${ }^{(1)}$-2012-054 emitió la escala de remuneración tanto para oficiales como para personal de tropa; salarios que van desde los 933 dólares para soldados hasta los 5.560 dólares para generales o almirantes.

En relación al sector de la educación, se la considera como uno de los pilares fundamentales para alcanzar desarrollo y crecimiento inclusivo (CEPAL/CAF/OCDE, 2014). Así entonces, se realizaron en el país una serie de esfuerzos y estrategias para mejorar su calidad; y, en este sentido, la mejora salarial fue vista como aliciente para alcanzar esta responsabilidad. En todo caso, esta reestructuración fue posible con la Ley Orgánica de Educación Intercultural -LOEI- (Asamblea Nacional, 2011). Sobre los salarios, éstos fueron fijados a partir del título profesional, tiempo de servicio y sobre los resultados obtenidos en los procesos de evaluación (Art. 111). El escalafón más básico es la categoría $\mathrm{J}$, aplicado a aquellos con título de bachiller y que ganaron el concurso de méritos y oposición en zonas de difícil acceso. Mientras tanto, el nivel $\mathrm{A}$ es aplicado a aquellos con título de tercer grado o universitario, con veinticuatro años de experiencia y que hayan aprobado los cursos de formación requeridos (Ministerio de Educacion, 2018). De acuerdo al incremento salarial declarado, para el año 2018, estos sueldos van desde los 675 hasta los 1.676 dólares.

De igual manera que en el caso anterior, la educación superior también fue reestructurada, a través de la Ley Orgánica de Educación Superior -LOES- promulgada en el año 2006 (Asamblea Nacional, 2010). En conjunto, y para este mismo fin, se crearon instituciones de contro como el Consejo de Educación Superior -CES- y el Consejo de Evaluación, Acreditación y Aseguramiento de la Calidad de la Educación Superior -CEEASSES-. El primero, tiene como objeto planificar, regular y coordinar; el segundo, ejerce la rectoría de la política pública para el aseguramiento de la calidad en el área. En el año 2012, una vez establecida la parametrización y categorización de las instituciones de educación superior -IES- se cerraron un total de catorce universidades, puesto que la intención fue depurar el sistema universitario. Cabe recalcar que la constitución de 2008 ya había planteado la creación en el país de un sistema educativo responsable y con nuevos desafíos estratégicos.

Con respecto a los salarios de estos docentes de las IES, fueron fijados de acuerdo al Reglamento de carrera y escalafón del profesor e investigador del Sistema de Educación Superior, el cual regula la categorización y sus sueldos (Consejo de Educación Superior, 2012). A partir de la reforma del año 2015, los sueldos de profesores titulares en los diferentes niveles, sea auxiliar, agregado o principal, sin considerar los cargos adicionales de autoridad, van desde los 1.676 hasta los 2.967 dólares; desde luego, existe un conjunto de requisitos de excelencia que se deben cumplir (Arts. 19-22). Complementariamente a esto, la LOES exigió a los profesores titulares principales el título de Doctor o 
PhD hasta octubre del año 2017, y quienes no lo cumplan perderán esta condición; a la vez, el artículo 14 de dicha ley establece con la misma fecha límite que las IES deberán contar con al menos el $70 \%$ de docentes con este título.

Bajo este contexto de reformas salariales generadas en el país surge la necesidad de conocer acerca de la rentabilidad de los diferentes niveles o estados de educación; en otras palabras, a través de este trabajo se pretende analizar el correspondiente efecto de la educación sobre el nivel de ingresos en el individuo dentro de Ecuador. Para ello, se parte del concepto teórico, desde ya antiguo, que mayores niveles de especialización son indispensables para el desarrollo, y que a mayor inversión educativa mayor será la rentabilidad (Mincer, 1974; Schultz, 1961). En todo caso, en una primera parte de este trabajo se describirán las variables nacionales de ingreso y escolaridad a manera de un breve diagnóstico. Por otro lado, se establecerá el aporte a la renta del individuo por cada año de escolaridad y sobre sus diferentes niveles; esto último a partir de modelos econométricos de ingresos mincerianos.

\section{Educación, salarios y rentabilidad}

Esta sección abordará, por un lado, un breve diagnóstico sobre la situación nacional en cuanto al promedio de escolaridad y al salario que percibe la población; por otro, analizará la rentabilidad que tiene la educación en el país y por cada uno de los niveles de escolaridad en base a modelos econométricos de regresión semilogarítmicos múltiples.

\section{Panorama nacional y diagnóstico}

Por lo general, cuando se habla de capital humano se pone en consideración las habilidades, conocimientos, educación e incluso las destrezas que poseen las personas (Patrinos, Ridao-Cano, \& Sakellariou, 2006). Aunque el capital humano y la educación no siempre estuvieron relacionados entre sí puesto que se concibieron por separado. En la actualidad, la educación se la establece como un elemento esencial dentro del primero (Neira, 1998); incluso, llega a explicar el nivel de ingresos percibidos por un individuo. Es así que la relación entre capital humano, educación e ingresos se convierte en todo un tema de investigación con carácter propio. Sin embargo, para que un individuo invierta en educación deberá sentirse motivado por algún factor, sea por ejemplo que en el futuro esta decisión le traiga esa mejora salarial. Así la educación del individuo es una forma de inversión dentro del propio individuo (Schultz, 1959, 1961).

Con respecto a Ecuador, se ha pasado por épocas de auge y riqueza, pero pocos gobiernos han priorizado la inversión en educación; aspectos que al final han imposibilitado alcanzar mayores niveles de educación y por tanto de desarrollo. Para contrarrestar tales deficiencias se establecieron principios fundamentales dentro del sistema educativo, los cuales se encuentran en el marco constitucional de 2008; en éste se estableció a la educación como un derecho a lo largo de la vida y un deber ineludible del Estado (Art.26). En todo caso, la Encuesta Nacional de Empleo, Desempleo y Subempleo -ENEMDU-, del mes de diciembre de 2017, permite conocer la realidad que vive el país, y sobre ello parte este breve diagnóstico nacional(2).

En relación a los años de escolaridad se presencia que el promedio nacional es de 10,2 años. Sin embargo, este indicador desagregado por las áreas geográficas del país muestra una gran brecha, siendo la escolaridad para el área rural de 7,5 años y para el área urbana de 11,3 años. Por su parte, la región insular tiene la cifra más alta con respecto a las demás con 12,4 años. De acuerdo al sexo, los hombres poseen un leve mayor nivel de escolaridad con 10,2 años frente a las mujeres (10,1 años).
Tabla 1. Promedio de escolaridad e ingresos en Ecuador Años de escolaridad y dólares US\$

\begin{tabular}{lcc}
\hline \multicolumn{1}{c}{ Descripción } & Escolaridad & Ingresos \\
\hline Por área & & \\
Área nacional & 10,2 & 486,85 \\
Área rural & 7,5 & 354,35 \\
Área urbana & 11,3 & 540,88 \\
Por región & & \\
Sierra & 10,3 & 516,06 \\
Costa & 10,1 & 457,71 \\
Amazónica & 10,0 & 466,03 \\
Insular & 12,4 & $1.377,94$ \\
Por sexo & & \\
Hombres & 10,2 & 526,68 \\
Mujeres & 10,1 & 421,81 \\
\hline
\end{tabular}

Fuente: Elaboración propia a partir de la información del Instituto Nacional de Estadística y Censos: ENEMDU 2017

Sobre los ingresos percibidos, a partir de la misma encuesta, se puede observar que en promedio los individuos ganan a nivel nacional 486,85 dólares. Ahora bien, en el área urbana el ingreso promedio es de 540,88 dólares; mientras que en el área rural es de 354,35 dólares. La región insular posee los mayores niveles de ingresos con 1.377,94 dólares. Acorde al sexo, el hombre (526,68 dólares) gana mucho más que la mujer (421,81 dólares).

\section{Metodología}

Después de haber revisado la literatura relevante acerca del capital humano y su relación con la rentabilidad en la educación y el diagnóstico estadístico de dichas variables en Ecuador, en este artículo para la determinación de la rentabilidad educativa se construirán modelos econométricos de ingresos mincerianos bajo dos metodologías.

En el primer modelo, la función de ingresos no presenta diferenciaciones en cuanto a los niveles de estudios, es decir, la variable escolaridad se presenta como una variable cuantitativa continua. Es así que la rentabilidad hallada es general y una sola por cada año de estudio adicional. En el segundo modelo se aplicaron variables dicotómicas para la diferenciación respectiva de los distintos niveles de estudios.

En relación al primer modelo (ecuación 1), Mincer (1974) propuso tres variables: ingresos $(\mathrm{Y})$, escolaridad $(\mathrm{S})$ y años de experiencia laboral que, al ser de difícil determinación, es sustituida por la edad (X). El estimador de la regresión del primer modelo $\beta_{-} 1$ equivaldría a la tasa de rentabilidad educativa; el antilogaritmo del intercepto $\beta \_0$ equivaldría al ingreso en ausencia de escolaridad y experiencia laboral; y $\varepsilon$ implica la variable de la perturbación estocástica. Cabe señalar que Mincer agrega las variables $X$ y $X^{2}$ para capturar la concavidad en la función de ingresos (Barceinas, 1999, pág. 105).

$\operatorname{Ln}(Y)=\beta_{0}+\beta_{1} S+\beta_{2} X+\beta_{3} X^{2}+\varepsilon$

En el segundo modelo (ecuación 2) se plantea la diferenciación de los niveles de instrucción educativa, en donde, $Y$ es el ingreso, A simboliza a los coeficientes de regresión para cada variable dicotómica según el nivel educativo (Dn), $X$ y $X^{2}$ representan a la edad y la edad al cuadrado junto con sus respectivos estimadores $\gamma_{1}$ y $\gamma_{2}$. Asíww entonces, una vez desarrollada la ecuación 2 con los cinco niveles descritos en la tabla 2. Posteriormente, se elimina la constante a fin de evitar el problema de multicolinealidad de las variables dicotómicas (Gujarati \& Porter, 2009, pág. 282). Bajo la consideración matemática de que la sumatoria de las cinco dicotómicas es igual a 1 se tiene que $D_{1}=1-D_{2}-D_{3}-D_{4}-D_{5}$.

Al sustituir $D_{1}$ en la ecuación 2 y luego de agrupar cada una de las variables dicotómicas $\left(D_{n}\right)$ se tiene por consiguiente la ecuación 3. No obstante, con el fin de tener una expresión más acorde a las formas econométricas se reemplazarán las diferencias con coeficientes betas (ecuación 4). 
$\operatorname{Ln}(Y)=a+\sum_{n=0}^{m} A_{n} \cdot D_{n}+\gamma_{1} \cdot X+\gamma_{2} \cdot X^{2}+\varepsilon$

$\operatorname{Ln}(Y)=A_{1}+\left(A_{2}-A_{1}\right) \cdot D_{2}+\left(A_{3}-A_{1}\right) \cdot D_{3}+\left(A_{4}-A_{1}\right) \cdot D_{4}+\left(A_{5}-A_{1}\right) \cdot D_{5}+\gamma_{1} \cdot X+\gamma_{2} \cdot X^{2}+\varepsilon$

$\operatorname{Ln}(Y)=\beta_{1}+\beta_{2} \cdot D_{2}+\beta_{3} \cdot D_{3}+\beta_{4} \cdot D_{4}+\beta_{5} \cdot D_{5}+\gamma_{1} \cdot X+\gamma_{2} \cdot X^{2}+\varepsilon$

Una vez especificados matemática y econométricamente los dos modelos de regresión, la estimación de los mismos permitirá medir el aporte al ingreso del individuo por cada año de escolaridad y en cada nivel de instrucción educativa.

\section{Resultados}

A través de este trabajo se determinó el efecto que tiene la educación sobre el nivel de ingresos en el individuo en Ecuador, a su vez, se presentó un breve diagnóstico sobre la realidad del país en cuanto a los años de escolaridad y el nivel de ingresos que perciben. No obstante, previo a conocer acerca de estas rentabilidades, se resalta la importancia de conocer el contexto histórico, y, por ello, se expusieron de manera resumida dentro de la introducción las diferentes reformas salariales que existieron desde el año 2008. Una vez presentados estos antecedentes se mostraron los diferentes análisis y cálculos, los cuales partieron de los datos expuestos por la ENEMDU del mes de diciembre de 2017. De esta manera, se generó el ya mencionado diagnostico nacional y, a continuación, se determinarán las rentabilidades para cada uno de los niveles educativos alcanzados. El cálculo de la rentabilidad educativa se basa en la construcción de dos modelos econométricos de regresión.

En primer lugar, la ecuación 5 muestra los coeficientes o parámetros del primer modelo de regresión estimado. Sobre los datos estimados en esta ecuación y, a partir de un modelo econométrico de ingresos minceriano, se observa que en promedio la educación incrementa la renta de un individuo en 9,91\% por cada año de escolaridad obtenido. Por su parte, cada año de experiencia laboral aumenta en promedio el ingreso de una persona en $6,06 \%$. Todo esto a partir de una función lineal en la que se plantea además que el individuo con ausencia de escolaridad y experiencia laboral recibe un ingreso de 27 dólares. Con todo, se puede concluir que un mayor nivel de instrucción educativa se traduce en mejores ingresos y, consecuentemente, una mejor calidad de vida.

$$
\operatorname{Ln}(\mathrm{Y})=\underbrace{3,30}_{(1.093,34)}+\underbrace{0,0606}_{(876,71)}(\mathrm{S})+\underbrace{0,0991}_{(724,39)}(\mathrm{X})-\underbrace{0,0011}_{(-723,45)}\left(\mathrm{X}^{2}\right)+\varepsilon
$$

Por su parte, la expresión 6 muestra los coeficientes o parámetros del segundo modelo de regresión estimado; sin embargo, de acuerdo a Barceinas (1999), las rentabilidades a hallarse parten de los coeficientes de regresión de $A_{n}$ y no a partir de las $\beta_{n}$. Así, luego de resolver las cinco expresiones $\left(\beta_{1}=A_{1} ; \beta_{2}=A_{2}-A_{1} ; \beta_{3}=A_{3}-A_{1} ; \beta_{4}=A_{4}-A_{1} ; \beta_{5}=A_{5}-A_{1}\right)$ se procederá a determinar las rentabilidades según la ecuación 7 , donde $r_{n}$ es la rentabilidad por cada nivel de instrucción educativa, con excepción del nivel 1, ya que, en éste no hay inversión por ausencia de escolaridad; $A_{n}$ son los coeficientes antes determinados; $y t_{n}$ son los años de escolaridad por cada nivel.

$$
\begin{aligned}
& \ln (\mathrm{Y})=\underbrace{3,945677}_{(4,28)}+\underbrace{0,5172357}_{(2,17)}\left(\mathrm{D}_{2}\right)+\underbrace{0,8864718}_{(2,23)}\left(\mathrm{D}_{3}\right)+\underbrace{1,439404}_{(2,28)}\left(\mathrm{D}_{4}\right)+\underbrace{2,223409}_{(3,61)}\left(\mathrm{D}_{5}\right)+ \\
& \underbrace{0,0544554}_{(2,73)}(\mathrm{X})-\underbrace{0,000639}_{-2,84}\left(\mathrm{X}^{2}\right)+\varepsilon \\
& r_{n}=\frac{\left(A_{n}-A_{n-1}\right)}{t_{m}}
\end{aligned}
$$

De esta manera, la rentabilidad para cada nivel educativo quedaría expresada de acuerdo a la siguiente fórmula: $r_{2}=\left(A_{2}-A_{1}\right) / 7 ; r_{3}=\left(A_{3}-\right.$ $\left.A_{2}\right) / 6 ; r_{4}=\left(A_{4}-A_{3}\right) / 5 ; r_{5}=\left(A_{5}-A_{4}\right) / 2$. Al reemplazar los factores encontrados de $A n$, estos cálculos tendrían los resultados porcentuales expuestos en la tabla 2: $r_{2}=(4,4629-3,9456) / 10=0,0517 ; r_{3}=(4,8321$ $4,4629) / 3=0,1231 ; \quad r_{4}=(5,3850-4,8321) / 5=0,1106 ; \quad r_{5}=(6,1691-$ $5,3851) / 2=0,3920$.
Tabla 2. Rentabilidad por cada nivel de educación

\begin{tabular}{ccccc}
\hline Nivel de educación & Descripción & \# de años por nivel & Ingresos & Rentabilidad \\
\hline Nivel 1 & Sin escolaridad & 0 & 182,86 & No existe \\
Nivel 2 & Educación básica & 10 & 333,53 & $5,17 \%$ \\
Nivel 3 & Educación media & 3 & 470,47 & $12,31 \%$ \\
Nivel 4 & Educación superior & 5 & 835,22 & $11,06 \%$ \\
Nivel 5 & Educación de posgrado & 2 & $1.758,28$ & $39,20 \%$ \\
\hline
\end{tabular}

Fuente: Elaboración propia a partir de la información del Instituto Nacional de Estadística y Censos: ENEMDU 2017

Si se segmenta a la educación en los cinco niveles establecidos por las normativas ecuatorianas, un individuo con posgrado (nivel cinco) cuenta con el mayor ingreso respecto al resto de niveles con 1.758,28 dólares en promedio, mientras que el individuo con ausencia de escolaridad (nivel uno) percibe 182,86 dólares. A partir de la construcción de un modelo econométrico de ingresos minceriano en el que se especifican los diferentes niveles de escolaridad con el uso de las variables dicotómicas, se exponen las diferentes rentabilidades resultantes. De esta manera se puede observar que la educación básica queda definida con una rentabilidad de 5,17\%; la educación media con 12,31\%; la educación superior alcanza un 11,06\%, mientras que para posgrado se tiene un 39,20\%. Cabe indicar que no existe rentabilidad calculada del individuo con ausencia de educación, ya que, no se realizó inversión alguna, con lo cual su rentabilidad será nula. Estos resultados presentados en el segundo modelo se complementan con los resultantes del primer modelo, mismos que revelaron que por cada año de escolaridad que incremente un individuo, en promedio, aportará a la renta de éste en un 6,06\%. De la misma manera, cada año de experiencia laboral que adquiera un individuo, en promedio, contribuirá a su renta en un 9,91\%.

\section{Conclusiones}

La teoría del capital humano hace referencia a la inversión y al esfuerzo que hace el ser humano para capacitarse, lo que promoverá un futuro con altos niveles de rentabilidad sobre dicha inversión. Esto expone, en todo caso, a la educación como un bien colectivo que debe ser universalizado para obtener una sociedad lista que enfrente al mundo actual. Por este motivo, la educación pasó de ser una adquisición de conocimientos y habilidades que dignifica al individuo a ser una pieza estratégica para el desarrollo económico y social de éste. De este modo, la educación y el empleo se han convertido en factores relevantes que han generado cambios drásticos en el mercado laboral.

En relación al diagnóstico, se pudo observar que el promedio de años de escolaridad en Ecuador es de 10,2 años. Pese a ello, si se analiza a este indicador territorialmente o se lo desagrega por grupos poblacionales se denota cierta dispersión en los resultados. En lo referente al nive de ingresos, los individuos a nivel nacional ganan en promedio 486,85 dólares. Estos resultados muestran una mayor disparidad, respecto a la escolaridad, si se los analiza por áreas geográficas, regiones o sexo. Sobre el cálculo de las rentabilidades, a través del uso de un modelo de ingresos general minceriano, se concluye que cada año de escolaridad aporta a la renta con el 6,06\%, mientras que por cada año de experiencia laboral se alcanza el 9,91\%. Por otro lado, según el modelo en el que se especifican los diferentes niveles de educación, el nivel de educación básica aporta al individuo con el 5,17\% en su renta; para los de educación media el 12,31\%; en educación superior con el 11,06\%; y posgrado con el $39,20 \%$.

A la luz de la información generada se recalca entonces lo que la teoría ya ha venido expresando con contundencia: la educación se convierte en un elemento esencial para mejorar el ingreso del individuo y, posiblemente, su bienestar económico. Desde una visión más global, ésta juega un papel decisivo para transformar a un país; ya que, una población correctamente instruida puede alcanzar una mayor productividad, lo cual se verá reflejado en el uso y generación de nuevas tecnologías, procesos y mejores productos. Es por este conjunto de razones que un gobierno deberá siempre esforzarse por alcanzar mayores niveles de calidad educativa a través de inversiones eficientes; y desde luego, no ceder ante presiones de ciertos grupos que buscan ablandar estos procesos por conveniencias particulares. Es importante por úl- 
timo mencionar que "la instrucción de las masas afianza la libertad y destruye la esclavitud" (Rocafuerte, 1835).

\section{Notas}

(1) Rafael Correa Delgado, Presidente Constitucional de la República de Ecuador durante el periodo 2007 a 2017, mediante Decreto Ejecutivo No. 500 del 26 de noviembre de 2014, modificó la denominación del Ministerio de Relaciones Laborales por Ministerio de Trabajo.

(2) Las cifras de los indicadores expuestos en el Panorama Nacional y Diagnóstico de la Educación, a nivel nacional y a nivel del resto de desagregaciones, se calcularon de la Encuesta Nacional de Empleo, Desempleo y Subempleo -ENEMDU- levantada por el Instituto Nacional de Estadística y Censos -INEC- en diciembre de 2017.

\section{Referencias}

Asamblea Nacional. (2011). Ley orgánica de educación intercultural. Registro oficial del Ecuador No 417, de 31-marzo-2011. Última reforma de 16-enero-2016, por Ley orgánica de servico público de energía eléctrica, publicada en el Tercer Suplemento del Registro Oficial No. 418. .

Asamblea Constituyente. (2008a). Mandato 8. Eliminación y prohibición de la tercerización, intermediación laboral, contratación laboral por horas, y cualquier forma de precarización de las relaciones de trabajo. Registro Oficial del Ecuador 330, 6-V-2008.

Asamblea Constituyente. (2008b). Constitución de la República del Ecuador. Registro Oficial 449 de 20-oct.-2008; última modificación: 21-dic.-2015.

Asamblea Nacional. (2010). Ley orgánica de educación superior. Registro Oficial del Ecuador, Suplemento 298 de 12-oct.-2010. Última modificación 30-dic.-2016.

Asamblea Nacional. (2015). Ley orgánica del servicio público. Registro oficial del Ecuador, Suplemento 294 de 06-octubre-2010. Última modificación 28-marzo-2016.

Barceinas, F. (1999). Función de ingresos y rendimiento de la educación en Mexico. EECO, 14(1).

CEPAL/CAF/OCDE. (2014). Perspectivas económicas de América Latina 2015. Educación, competencias e innovación para el desarrollo. Santiago de Chile. doi:http://dx.doi.org/10.1787/leo-2015-es

Congreso Nacional. (2005). Código de trabajo. Registro Oficial del Ecuador, Suplemento 167 de 16-dic-2005. Última modificación por Asamble Nacional 28-marzo-2016. Incluye Ley Orgánica para la promoción del trabajo juvenil, regulación de la jornada de trabajo, cesantía y seguro de desempleo. Recuperado el 09 de septiembre de 2017, de http://www.ecuadorlegalonline.com/laboral/ codigo-de-trabajo/

Consejo de Educación Superior. (2012). Reglamento de Carrera y Escalafón del Profesor e Investigador del Sistema de Educación Superior. Resolución del Pleno Consejo No RPC-SO-037-No.265-2012.

Gujarati, D., \& Porter, D. (2009). Econometria. (T. d. edición, Trad.) MéxiCO, D.F.: MCGRAW-HILLINTERAMERICANA EDITORES, S.A. DE C.V.

Mincer, J. (1974). Schooling, Experience and Earnings. Columbia University Press.

Ministerio de Educacion. (2018). Escalafón del magisterio nacional y sus categorías. Recuperado el 02 de septiembre de 2017, de Escalafón del magisterio nacional y sus categorías: https://educacion.gob.ec/recategorizacion/

Ministerio de Trabajo. (2017). Acuerdo Ministerial No MDT-2017-0301. Recuperado el 09 de septiembre de 2017, de http://www.trabajo.gob.ec/ wp-content/uploads/downloads/2017/01/ACUERDO-NO.MDT-2016-0301.pdf Ministerio de Trabajo. (01 de 12 de 2017). http://www.trabajo.gob.ec. Recuperado el 18 de Agosto de 2017, de Ministerio de Trabajo: http://Www.trabajo.gob.ec/ministerio-del-trabajo-aclara-a-los-servidores-publicos-de-carrera/

Neira, I. (1998). Modelos econometricos de capital humano: Principales enfoques y evidencia empirica. Working Paper Series Economic Developmen. Recuperado el 15 de julio de 2017, de https://www. researchgate.net

Patrinos, H. A., Ridao-Cano, C., \& Sakellariou, C. (2006). Estimating the returns to education: accounting for heterogeneity in ability. Policy Research Working Paper, 1-38. Obtenido de https://doi.org/10.1596/1813-9450-4040

Rocafuerte, $V$. (1835). Discurso con motivo de concluir las sesiones de la convención constituyente que sancionara la constitución de 1845, en la ciudad de Cuenca, Ecuador. Recuperado el 10 de septiembre de 2017, de http://constitucionweb.blogspot.com/2010/04/discurso-de-vicente-rocafuerte-en-la.htm/

Schultz, T. (1959). Investment in man: an economist view. The Social Service Review Vol. XXXIII, 109-117.

Schultz, T. (1961). Investment in Human Capital. En American Economic Review, 51(No 1), 1-17. 